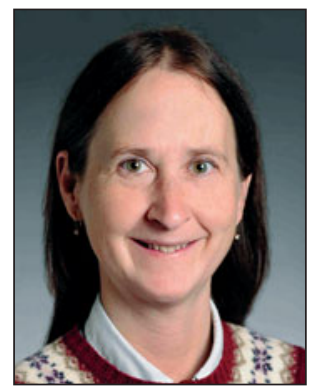

Sara A. Majetich

Guest Editor for this issue of MRS Bulletin

Carnegie Mellon University, Pittsburgh,

PA, USA; tel. 412-268-3105;

and email sara@cmu.edu.

Majetich is a professor in the Physics Department at Carnegie Mellon University. She received her $\mathrm{PhD}$ degree in condensed matter physics from the University of Georgia, and then worked at Cornell University as a postdoc. Her research interests focus on magnetic nanoparticles and nanostructures-including both materials preparation and nanoscale characterization-for potential applications in data storage media, biomedicine, high frequency inductors, and permanent magnets. She has authored over 150 papers and has three patents. She has received a National Young Investigator Award from the US National Science Foundation, was a 2007 Distinguished Lecturer of the IEEE Magnetics Society, and is a Fellow of the American Physical Society.

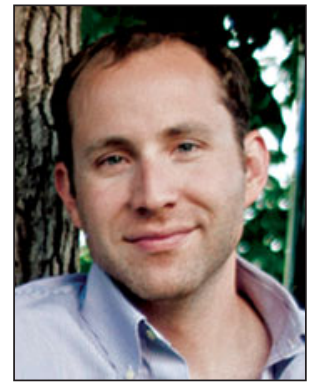

\section{Thompson Mefford}

Guest Editor for this issue of MRS Bulletin

Clemson University, Clemson,

SC, USA; tel. 864-656-4307; and

email Mefford@clemson.edu.

Mefford is an assistant professor in the Department of Materials Science and Engineering at Clemson University. He earned his PhD degree in macromolecular science and engineering in 2007 from Virginia Tech and worked at The Ohio State University as a postdoc. His research focuses on developing stable, biocompatible, polymer-metal oxide nanoparticle complexes and composites for biomedical, energy, and environmental applications. These include developing materials for magnetic hyperthermia, measuring the colloidal stability of MRI contrast agents, and investigating the environmental fate of nanomaterials.

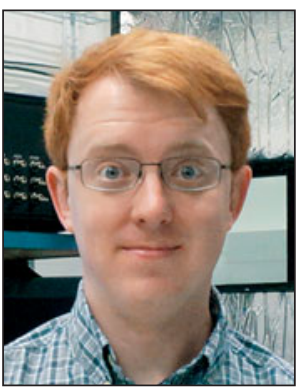

\section{Jacob Alldredge}

National Institute of Standards and Technology, Boulder, CO, USA; tel. 303-497-4386; and email alldredg@boulder.nist.gov. Alldredge graduated from the University of Florida in 2001 with a bachelor's degree in physics. He then received a NAF-Fullbright Fellowship to work for a year in the Netherlands for Cees Dekker at Delft University of Technology. He graduated from Cornell University with a PhD degree in physics in 2008 developing scanning tunneling microscopy for studying superconducting materials followed by a postdoc at the University of Colorado studying the local density of states in cuprates. He currently is working at NIST developing magnetic particle force microscopy.

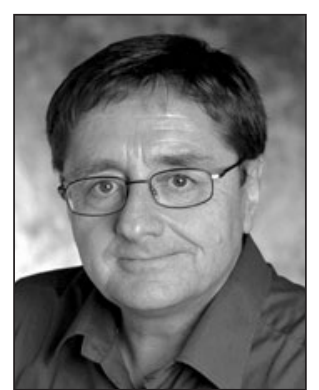

\section{Roy Chantrell}

Department of Physics, The University of

York, York, UK; tel. +44 (0) 1904322253

and email roy.chantrell@york.ac.uk.

Chantrell is a professor in the Department of Physics at The University of York. Prior to his current chair, he was director at Seagate Research (Pittsburgh). His research interests are in the development of theoretical and computational modeling of magnetic materials. His group is currently developing multiscale models linking ab initio, atomistic, and mesoscopic approaches. $\mathrm{He}$ is editor of the Journal of Magnetism and Magnetic Materials. He is a Fellow of the IEEE and of the American Physical Society. He has published around 400 papers and has presented numerous invited papers at magnetism meetings.

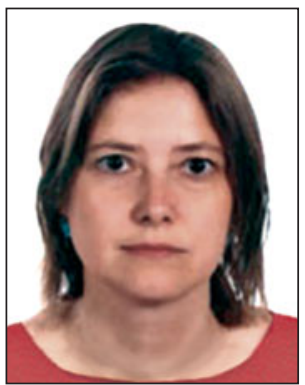

Oksana Chubykalo-Fesenko

Instituto de Ciencia de Materiales de Madrid, CSIC, Madrid, Spain; tel. +34-91-3349054; and email oksana@icmm.csic.es.

Chubykalo-Fesenko is a senior researcher at the Institute of Material Science of Madrid, Spanish National Research Council (CSIC). She received her $\mathrm{PhD}$ degree in condensed matter theory at Kharkov State University, Ukraine. She then worked at the University of Oxford (UK), Complutense University (Madrid, Spain), University of Como (Italy), University of the Basque Country (Spain), and the IBM Almaden Research Center (San Jose, USA). Her research interests include different aspects of the theory of nanomagnetism, including advanced micromagnetics of magnetic nanostructures, atomistic models, thermal effects in nanoparticles and nanostructures, and magnetization dynamics. She has authored around 130 articles in research journals.

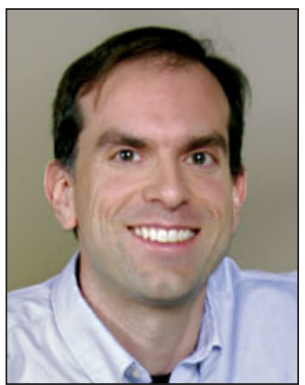

Thomas M. Crawford

University of South Carolina, Columbia,

SC, USA; tel. 803-777-7151; and

email crawford@physics.sc.edu.

Crawford is an associate professor in the Physics Department at the University of South Carolina. He received his $\mathrm{PhD}$ degree in condensed matter physics from the University of Colorado at Boulder, and after being an NRC postdoc at NIST-Boulder, spent more than six years at Seagate Technology as a research staff member. His research interests focus on nanomagnetism, specifically advanced magnetic measurements, recently employing magnetic recording for nanomanufacturing advanced materials from magnetic nanoparticles. He has authored over 40 papers and holds 11 patents. He is a winner of the University of South Carolina's Michael J. Mungo undergraduate teaching award and in 2013 was recognized as a "Rising Star" by the university's office of research.

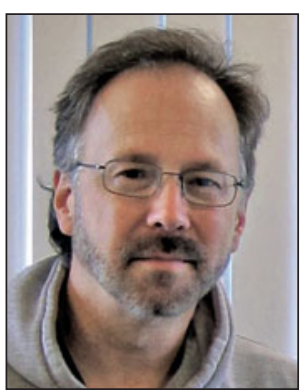

\section{Jon Dobson}

J. Crayton Pruitt Family Department of Biomedical Engineering, Department of Materials Science and Engineering, \& Institute for Cell Engineering and Regenerative Medicine, University of Florida, Gainesville, FL, USA; tel. 352-2739222; and email jdobson@ufl.edu.

Dobson is a professor of Biomedical Engineering and Biomaterials and Director of the Institute for Cell Engineering and Regenerative Medicine (ICERM) at the University of Florida. He received his $\mathrm{PhD}$ degree in natural sciences from the Swiss Federal Institute of Technology, ETH-Zurich. His research focuses on biomedical applications of micro- and nanoparticles and includes studies of nanomagnetic actuation of cell surface receptors for cell engineering and regenerative medicine, nanomagnetic gene transfection, iron biomineralization related to neurologica disorders, magneto-microfluidics and MNP harvesting technologies for disease diagnosis and treatment, and development of novel magnetic actuation systems for bioMEMS devices. He has authored over 120 papers and has nine granted patents. He has received a Royal Society of London Wolfson Research Merit Award (2004) and is Fellow of the Institute of Nanotechnology and the Society of Biology.

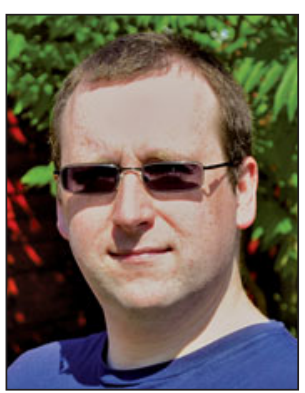

\section{Richard Evans}

Department of Physics, The University of York, York, United Kingdom; tel. +44 (0)1904 322254; and email richard.evans@york.ac.uk. Evans is currently a post-doctoral research fellow at the University of York, where he also obtained his $\mathrm{PhD}$ degree in 2008. His research has centered on understanding the effects of atomic structure and disorder on the physical properties of magnetic materials, ultrafast magnetism, and heat-assisted magnetic recording. He is also the lead developer of the open source VAMPIRE 
software package for parallel atomistic simulations of magnetic materials. He has published over 25 refereed journal articles and has given invited talks at international and local specialist magnetic meetings, as well as several contributed talks at international conferences.

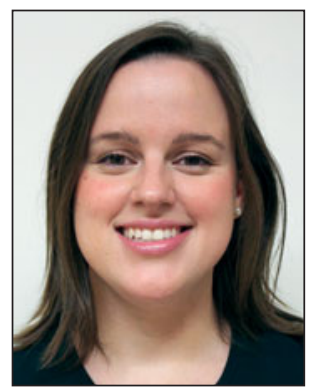

Bettina Kozissnik

University of Florida, Gainesville, FL, USA; tel.352-273-3995; and email b.kozissnik@ufl.edu. Kozissnik is a postdoc at the University of Florida. She earned her PhD degree under the guidance of Kerry Chester and Quentin Pankhurst at the University College London in co-operation with Davy-Faraday Research Laboratories at The Royal Institution of Great Britain. Her current research focuses on improving the diagnosis of osteoarthritis using magnetic nanoparticles, and magnetically mediated energy delivery.

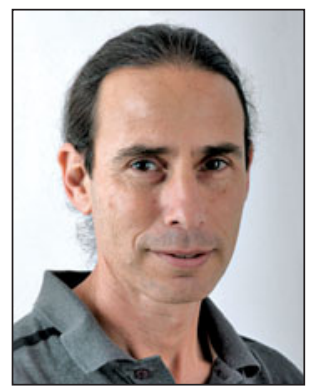

\section{Gil Markovich}

School of Chemistry, Tel Aviv

University, Israel; tel. 972-3-6406985;

and email gilmar@post.tau.ac.il.

Markovich is a professor in the Department of Chemical Physics, School of Chemistry, Tel Aviv University, and is also the chairman of the School of Chemistry. He received his BSc (1989) and PhD (1996) degrees from Tel Aviv University and spent two years as a postdoc at UCLA. In 1998, he established a laboratory for studies of colIoidal nanomaterials at Tel Aviv University. His research interests include colloidal nanocrystals, nanomagnetism, nanoferroelectricity, and nano-chirality.

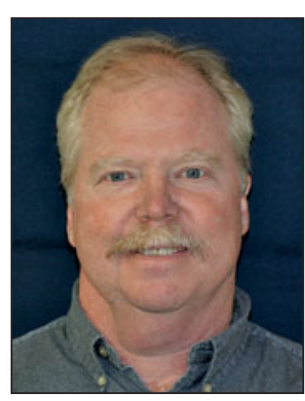

\section{John Moreland}

National Institute of Standards and Technology, Boulder, CO, USA; tel. 303-497-3641; and email moreland@boulder.nist.gov.

Moreland leads the Microsystems Project in the Physical Measurement Laboratory at the National Institute of Standards and Technology (NIST). He earned his BS degree in physics and chemistry at the University of Idaho and his PhD degree in physics at the University of California, Santa Barbara. Moreland was an IEEE Fellow in 2011. He has focused his efforts toward developing micro-electromechanical systems for metrology applications. Currently, the project is developing new contrast agents with better sensitivity, spatial resolution, and functionality for magnetic resonance imaging.

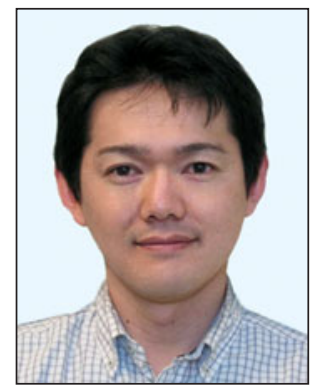

Yoshihiro Nakashima

National Institute of Standards and

Technology, Boulder, CO, USA; email

yoshihiro.nakashima@nist.gov.

Nakashima is a research postdoctoral student at Kyushu University and a guest researcher at the National Institute of Standards and Technology (NIST) since 2010. He studied electrical engineering at Kyushu University, Japan, and received his $\mathrm{PhD}$ degree in the area of magnetic shielding systems in 2010. His current research interests include characterization of magnetic particles for NMR and MRI bio-imaging and bio-assay applications.

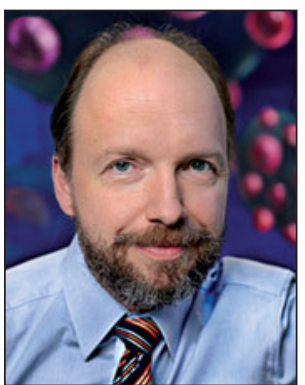

Stefan Odenbach

Chair of Magneto-fluid Dynamics,

Measuring, and Automation Technology,

Technische Universität Dresden, Germany;

tel. +49-351463-32062; and email

stefan.odenbach@tu-dresden.de.

Odenbach is a professor in the Department of Mechanical Engineering at TU Dresden. $\mathrm{He}$ earned his $\mathrm{PhD}$ degree in physics in 2007 from the Ludwig Maximilians University in Munich. He also worked at the University in Wuppertal as a postdoc and as an assistant professor at the University of Bremen. His research focuses on the fluid mechanics of magnetic hybrid materials, rheology, and measuring technology. A special focus of these investigations is the rheology of magnetic suspensions and elastomers and clarification of the microstructural reasons for the rheological properties of the materials in a magnetic field.

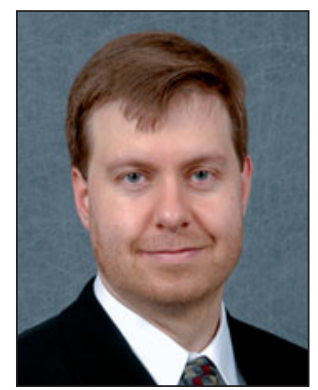

Joseph B. Tracy

North Carolina State University,

Raleigh, NC, USA; tel. 919-513-2623

and email jbtracy@ncsu.edu.

Tracy is an assistant professor in the Department of Materials Science and Engineering at North Carolina State University. He received his BS degree in chemistry from the University of California, Santa Barbara, and his PhD degree in physical chemistry from MIT, followed by postdoctoral studies at the University of North Carolina, Chapel Hill. He joined NC State in 2007 where his research interests include the synthesis, self-assembly, and transformations of metal and magnetic nanoparticles. In 2011, he received an NSF CAREER Award.

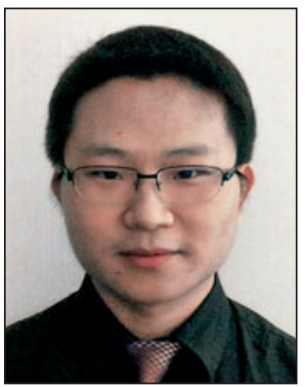

Tianlong Wen

Carnegie Mellon University, Pittsburgh,

PA, USA; tel. 412-268-2319; and

email halong@andrew.cmu.edu.

Wen has been a postdoctoral research associate in the Physics Department at Carnegie Mellon University since 2011 . He received his $\mathrm{PhD}$ degree in materials science and engineering from the University of Washington in 2010. His dissertation focused on magnetic, thermal, and electrical properties of cobalt-based magnetic nanocomposites. His current research focus is to fabricate large-area self-assembled monolayers of nanoparticles and use them as nanomasks to fabricate dense hole and pillar arrays with a feature size $\sim 10 \mathrm{~nm}$.

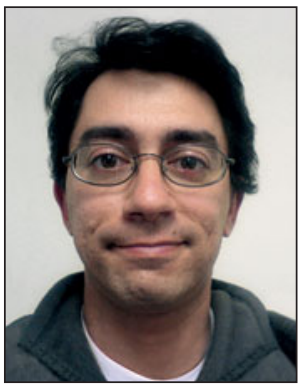

Gary Zabow

National Institute of Neurological Disorders and Stroke, Bethesda, MD, USA; tel. 303-4974657; and email zabow@boulder.nist.gov. Zabow is a senior research fellow at the National Institutes of Health (NIH) and a concurrent guest researcher at the National Institute of Standards and Technology (NIST). He earned a PhD degree from Harvard University. His research interests range widely and include atomic physics, microfluidics, magnetism, magnetic resonance imaging (MRI), and micro- and nanofabrication techniques. His current research focuses on ways to leverage microfabrication technologies to enhance MRI functionality. 


\section{Kurt J. Lesker}

\section{Hydra Cool ${ }^{\text {TM }}$}

Revolutionary Water

Cooling Method

- Technically superior method of water cooling

- High ratio of cooling area to cost

- Utilizes radius bends, improving water flow

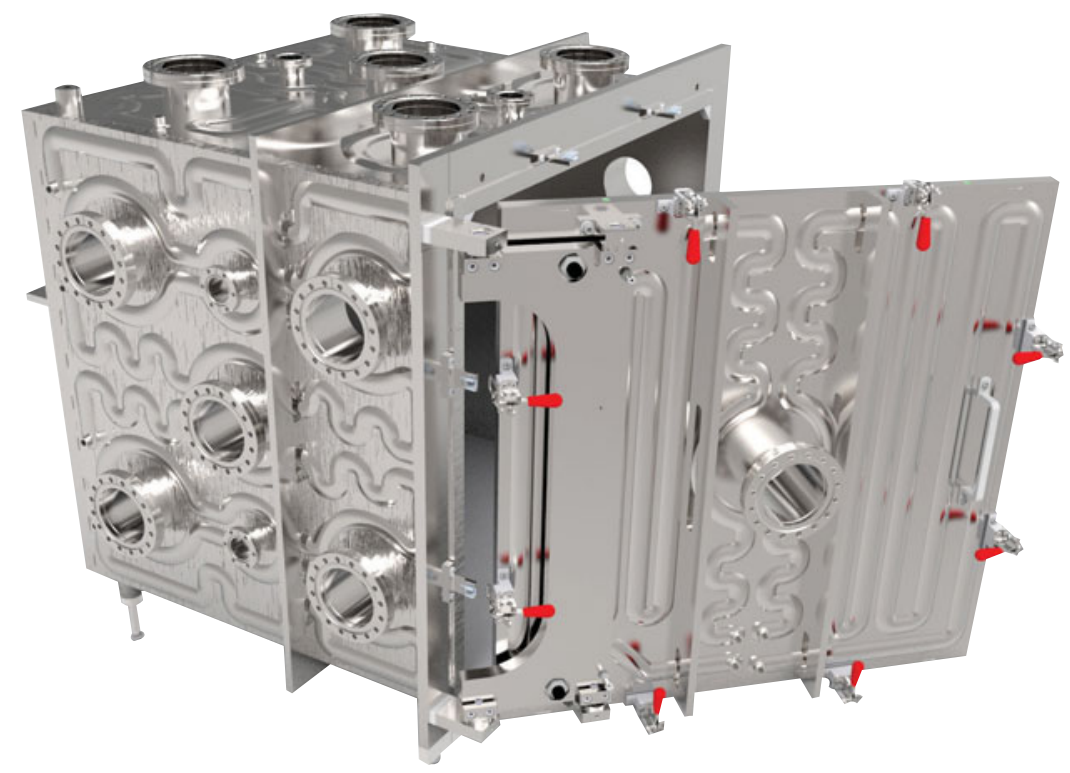

\section{Online Custom Product Creators}

\section{Chamber Builder ${ }^{\mathrm{TM}}$}

- Modify our standard chambers to create a product that fits your application.

- Get a price and interactive model in only a few minutes.
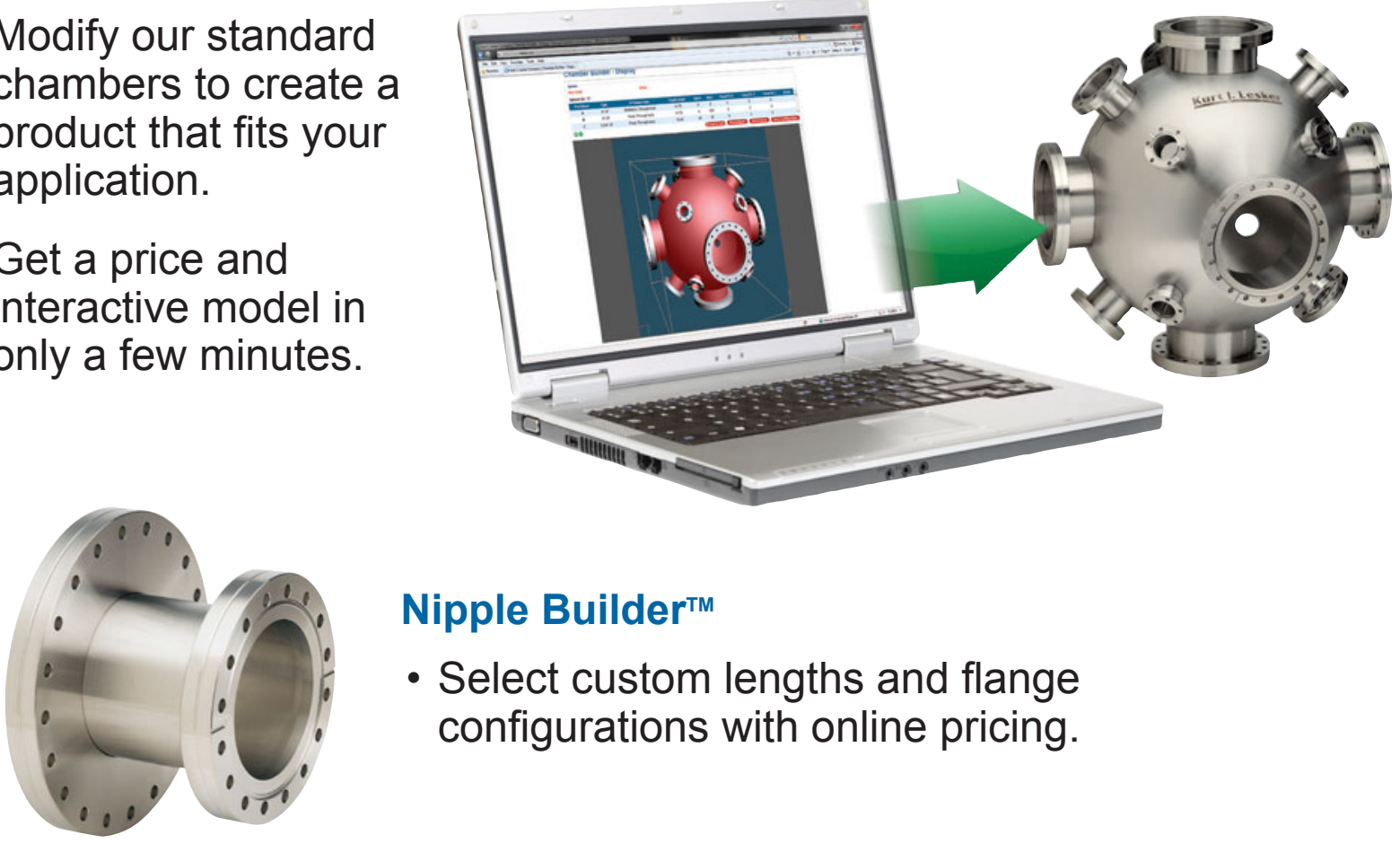

Nipple Builder ${ }^{\mathrm{TM}}$

- Select custom lengths and flange configurations with online pricing.

30 Years of In-house Manufacturing Experience
MANUFACTURING DIVISION

\section{www.lesker.com}


